\title{
構造系とゲインスケジュールドコントローラの統合化設計*
}

\author{
平 元 和 彦*1, 中 道 健 $太^{* 2}$
}

\section{An Integrated Design of Structural and Gain Scheduled Control Systems}

\author{
Kazuhiko HIRAMOTO*3 and Kenta NAKAMICHI \\ ${ }^{* 3}$ Department of Mechanical Engineering, Akita University, \\ 1-1 Tegata-gakuen-machi, Akita-shi, Akita, 010-8502 Japan
}

\begin{abstract}
We deal with an integrated design problem of structural and gain scheduled control systems for LPV systems. The integrated design problem is formulated as an optimization problem of structural design parameters and a gain scheduled controller minimizing $\mathscr{L}_{2}$ gain of the closed-loop system. Even in the simplest case the integrated design problem is represented as a BMI problem which cannot be solved exactly and efficiently. In the present paper we propose an iterative LMI-based method to solve the formulated optimization problem approximately. We can guarantee the local convergence of the closed-loop $\mathscr{L}_{2}$ gain with the proposed design algorithm even if the coefficient matrices of the state-space form of the control object are nonlinear functions on the structural design parameters. We show the effectiveness of the proposed design method through a design example.
\end{abstract}

Key Words: Integrated Design of Structural and Control Systems, LPV Systems, Gain Scheduled Control, LMI, Robust Control, Motion Control, Computer Aided Design

\section{1. 緒言}

従来, 制御系設計は, 制御対象の設計の後コント ローラを設計する二段階の手順で行われてきた. 多く の場合，制御系の性能の改善は，固定された制御対象 （のモテル）に対してコントローラ中に存在する設計 変数を調整することによって行われてきた. 制御系全 体の性能向上を目指す立場からは，前述のように制御 対象とコントローラをそれぞれ別個に設計することは 合理的でない. 制御系の性能は, コントローラの設計 変数だけでなく, 物理パラメータなどの制御対象中の 変数にも明らかに強く依存しているからである.

この考え方に基づき, 制御対象中の設計変数とコン トローラを閉ループ系の性能を指標として同時に最適 設計し, より高い制御性能を達成することを目指す構 造系と制御系の統合化設計に関する研究が行われてき た(1) (3)など. これらの研究報告例で提案または紹介さ れている設計手法のほとんどは, いずれも制御対象が LTI (Linear Time Invariant) モデルで表現できるという 前提条件の下で構築された数值的な繰り返し最適化ア

* 原稿受付 2005 年 4 月 4 日.

*1 正員, 秋田大学工学資源学部機械工学科( $\mathbf{\omega}$ 010-8502 秋田 市手形学園町 1-1)

*2 准員, (株) 沖データシステムズ (- 960-2153 福島市庄野字 立田 1-1).

E-mail : hira (a ipc.akita-u.ac.jp
ルゴリズムであった.

ロボットマニピュレータや航空・宇宙分野の応用で は, 制御対象の動特性がある検出可能な時変パラメ一 タ（スケジューリングパラメータ）に依存して変化す る場合が多い. 制御対象の線形状態方程式の係数行列 が, スケジューリングパラメータの関数で表されるシ ステムは LPV (Linear Parameter Varying) システムと 呼ばれている. LPV システムは, 非線形システムの一 部のクラスも高い精度で近似可能であり, その記述能 力は非常に高い. さらに, LPV システムに対する LMI に基づくゲインスケジュールドコントローラの設計手 法が, 近年急速に整備されてきている ${ }^{(4) \sim(7)}$. 現在も, LPV システムの解析や制御系設計に関する研究が活発 に行われているが, LPV システムを制御対象として構 造系とコントローラを統合的に最適設計する研究は見 当たらない.

本論文では, 従来 LTI システムの制御系設計のみに ついて研究されてきた構造系と制御系の統合化設計を LPV システムに抎張する. 制御手法には, Apkarian ら ${ }^{(5)}$ によって提案されている LMI に基づくゲインスケ ジュールド制御則を採用する. このコントローラ設計

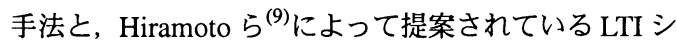
ステムに対する反復 LMI 近似を用いた統合化設計法 を組み合わせ, 局所的に最適な制御対象中の設計変数 
とゲインスケジュールドコントローラを求めるための 繰り返し設計アルゴリズムを提案する. 本手法では, 文献 ${ }^{(9)}$ と同様に, 制御対象の状態方程式の係数行列が 設計変数である構造系の質量, 隇衰, 剛性や状態方程 式の保数行列を唯一に定める基底変数の非線形関数之 なる場合においても設計結果の局所的最適性が保証さ れる.

本論文の構成を以下に示す，第 2 章では，対象とす る LPV システムとゲインスケジュールドコントロー ラの定義を行い, LPV システムに対する統合化設計問 題を定式化する. 第 3 章では, 定式化された統合化設 計問題に対する設計アルゴリズムを提案する．第 4 章 では，設計例として回転型倒立振子を取り上げ，提案 する統合化設計手法の有効性を示す. 第 5 章で, 本論 文のまとめを行い, 今後の課題を述べる.

本論文では, $t, g,{ }^{T}, I, \sigma_{\max }(A)$ および $\operatorname{det} A$ を時刻, 重力加速度, 行列の転置, 単位行列, 行列 $A$ の最大 特異值および行列式とする. また, $\mathscr{R}^{m}, \mathscr{R}^{m \times n}$ および $\mathscr{S}^{m}$ をそれぞれ $m$ 次元実べクトルの集合, $m$ 行 $n$ 列の 実行列の集合および $m$ 次元実対称行列の集合とする.

\section{LPV システムに対する統合化設計問題}

2.1 制御対象およびコントローラの定義 以下 の状態方程式で表される制御対象である LPV システ ム $P$ と, フルオーダのゲインスケジュールドコント ローラ $K$ を考える.

$P:\left\{\begin{aligned} \dot{x}(t)= & A(\alpha(t), \beta) x(t)+B_{1}(\alpha(t), \beta) w(t) \\ & +B_{2}(\alpha(t), \beta) u(t) \\ z(t)= & C_{1}(\alpha(t), \beta) x(t)+D_{12}(\alpha(t), \beta) u(t) \\ y(t)= & C_{2}(\alpha(t), \beta) x(t)+D_{21}(\alpha(t), \beta) w(t)\end{aligned}\right.$

$K:\left\{\begin{array}{l}\dot{x}_{K}(t)=A_{K}(\alpha(t), \dot{\alpha}(t)) x_{K}(t)+B_{K}(\alpha(t), \dot{\alpha}(t)) y(t) \\ u(t)=C_{K}(\alpha(t), \dot{\alpha}(t)) x_{K}(t)\end{array}\right.$

ここで, $x(t) \in \mathscr{R}^{n_{x}}, w(t) \in \mathscr{R}^{n_{w}}, u(t) \in \mathscr{R}^{n_{u}}, z(t) \in$ $\mathscr{R}^{n_{z}}$ および $y(t) \in \mathscr{R}^{n_{y}}$ は，それぞれ状態べクトル，外 乱, 操作量, 制御出力および観測出力である. $x_{K}(t) \in$ $\mathscr{R}^{n_{x}}$ はコントローラの状態ベクトルである. $\alpha(t):=$ $\left[\alpha_{1}(t), \ldots, \alpha_{n_{\alpha}}(t)\right]^{T} \in \mathscr{R}^{n_{\alpha}}$ は, 少なくとも $t$ で一回微 分可能なスケジューリングパラメータである. $\alpha(t)$ お よび $\dot{\alpha}(t)$ はそれぞれ以下の集合 $\mathscr{A}, \mathscr{D}_{\alpha}$ に属している とする.

$$
\begin{aligned}
\alpha(t) \in \mathscr{A}:=\left\{\alpha(t) \in \mathscr{R}^{n_{\alpha}} \mid \underline{\alpha} \leq \alpha(t) \leq \bar{\alpha}\right\}, \\
\underline{\alpha}:=\left[\underline{\alpha}_{1}, \ldots, \underline{\alpha}_{n_{\alpha}}\right] \in \mathscr{R}^{n_{\alpha}}, \bar{\alpha}:=\left[\bar{\alpha}_{1}, \ldots, \bar{\alpha}_{n_{\alpha}}\right] \in \mathscr{R}^{n_{\alpha}}, \\
\dot{\alpha}(t) \in \mathscr{D}_{\alpha}:=\left\{\dot{\alpha}(t) \in \mathscr{R}^{n_{\alpha}} \mid \underline{\delta} \leq \dot{\alpha}(t) \leq \bar{\delta}\right\}, \quad \text { (4) } \\
\underline{\delta}:=\left[\underline{\delta}_{1}, \ldots, \underline{\delta}_{n_{\alpha}}\right] \in \mathscr{R}^{n_{\alpha}}, \bar{\delta}:=\left[\bar{\delta}_{1}, \ldots, \bar{\delta}_{n_{\alpha}}\right] \in \mathscr{R}^{n_{\alpha}}
\end{aligned}
$$

$\beta \in \mathscr{R}^{n_{\beta}}$ は, 制御対象中の調整可能な設計変数をべク トルにまとめたものであり，以下のような集合 $\mathscr{B} に$ 属しているとする.

$$
\beta \in \mathscr{B}:=\left\{\beta \in \mathscr{R}^{n_{\beta}} \mid \underline{\beta} \leq \beta \leq \bar{\beta}, \underline{\beta}, \bar{\beta} \in \mathscr{R}^{n_{\beta}}\right\}
$$

なお，本論文では，一般化プラント $P$ の状態方程式 (1) の倸数行列が $\alpha(t) \in \mathscr{A}$ および $\beta \in \mathscr{B}$ の線形関数に限 定されないことに注意しておく.

2.2 統合化設計問題の定式化 式 (1) および (2) からなる閉ループ系 $G$ は，以下のようになる.

$$
G:\left\{\begin{array}{l}
\dot{x}_{G}(t)=A_{G} x_{G}(t)+B_{G} w(t) \\
z(t)=C_{G} x_{G}(t)
\end{array}\right.
$$

$$
\begin{gathered}
x_{G}(t):=\left[\begin{array}{c}
x(t) \\
x_{K}(t)
\end{array}\right], A_{G}(\alpha(t), \beta):=\left[\begin{array}{cc}
A & B_{2} C_{K} \\
B_{K} C_{2} & A_{K}
\end{array}\right], \\
B_{G}(\alpha(t), \beta):=\left[\begin{array}{c}
B_{1} \\
B_{K} D_{21}
\end{array}\right], C_{G}(\alpha(t), \beta):=\left[C_{1} D_{12} C_{K}\right]
\end{gathered}
$$

本論文では, すべての $\alpha(t) \in \mathscr{A}, \dot{\alpha}(t) \in \mathscr{D}_{\alpha}$ の下で, 閉 ループ系 $G$ の内部安定性を保ちながら次式の不等式 中の $\gamma>0$ を最小化するゲインスケジュールドコント ローラ $K$ を設計する.

$$
\int_{0}^{T_{f}} z^{T}(t) z(t) d t \leq \gamma^{2} \int_{0}^{T_{f}} w^{T}(t) w(t) d t, \forall T_{f} \geq 0
$$

式 (7) $\gamma>0$ は, 式 (6) の閉ループ系 $G$ の $\mathscr{L}_{2}$ ゲインで ある. 本論文では, 閉ループ系の $\mathscr{L}_{2}$ ゲイン $\gamma$ を制御 系の性能を測る指標とし, 構造系とゲインスケジュー ルド制御系の設計問題を以下のように定式化する.

構造系とゲインスケジュールド制御系の統合化 設計問題：式(1)の一般化プラントに対し, 式 (3) および (4)の条件下で, 式 (7) 中の $\gamma>0$ を 最小化するゲインスケジュールドコントローラ $K_{o p t}$ （式 (2)）および構造系の設計変数べクトル $\beta_{o p t} \in \mathscr{B}$ を求めよ.

\section{3. 設 計 手 法}

3.1 コントローラの設計法 式(1)で与えられる LPV システムに対して, 式 (2) のゲインスケジュール ドコントローラ $K$ を Apkarian らによって提案されて いる設計手法 ${ }^{(5)}$ を用いて, 以下の手順で求める.

Step 1: 集合 $\mathscr{A}$ から有限個数の部分集合 $\mathscr{A}^{g}:=$ $\left\{\alpha_{g}^{1}, \ldots, \alpha_{g}^{n_{g}}\right\}, \alpha_{g}^{i} \in \mathscr{A} \forall i$ を選択する. $n_{g}$ は, 設計者 によって決定される．この選択の過程をグリッディ ングという. 集合 $\mathscr{D}_{\alpha}$ を $n_{\alpha}$ 次元の超直方体と考え, その頂点を $\alpha_{d}^{i} \in \mathscr{R}^{n_{\alpha}}, i=1, \ldots, 2^{n_{\alpha}}$ とおく. 集合 $\mathscr{D}_{\alpha}^{g}:=\left\{\alpha_{d}^{1}, \ldots, \alpha_{d}^{n_{d}}\right\}, n_{d}:=2^{n_{\alpha}}$ を定義する. 
Step 2: 以下の $\alpha(t)$ に依存する行列変数を定義する.

$$
\begin{aligned}
X(\alpha) & :=X_{0}+\sum_{i=1}^{n_{p}} \bar{p}_{i}(\alpha) X_{i}, X_{j} \in \mathscr{S}^{n_{x}}, \\
Y(\alpha) & :=Y_{0}+\sum_{i=1}^{n_{p}} \bar{p}_{i}(\alpha) Y_{i}, Y_{j} \in \mathscr{S}^{n_{x}}, \\
\hat{A}(\alpha) & :=\hat{A}_{0}+\sum_{i=1}^{n_{p}} \bar{p}_{i}(\alpha) \hat{A}_{i}, \hat{A}_{j} \in \mathscr{R}^{n_{x} \times n_{x}}, \\
\hat{B}(\alpha) & :=\hat{B}_{0}+\sum_{i=1}^{n_{p}} \bar{p}_{i}(\alpha) \hat{B}_{i}, \hat{B}_{j} \in \mathscr{R}^{n_{x} \times n_{y}}, \\
\hat{C}(\alpha) & :=\hat{C}_{0}+\sum_{i=1}^{n_{p}} \bar{p}_{i}(\alpha) \hat{C}_{i}, \hat{C}_{j} \in \mathscr{R}^{n_{u} \times n_{x}}, \\
\forall j & =0, \ldots, n_{p}
\end{aligned}
$$

ここで, $\bar{p}_{i}(\alpha)\left(i=1, \ldots, n_{p}\right)$ は, 設計者によって指定 される $t$ に関して少なくとも一回微分可能な $\alpha(t)$ の スカラ関数である.

Step 3: $i=1, \ldots, n_{g}, j=1, \ldots, n_{d}$ に対して，以下の連 立 LMI の下で, $\gamma>0$ を最小化するような行列変数 $X(\alpha), Y(\alpha), \hat{A}(\alpha), \hat{B}(\alpha), \hat{C}(\alpha)$ を求める.

$$
\begin{aligned}
& E(i, j)<0, \\
& E(i, j):=\left[\begin{array}{cccc}
E_{11}(i, j) & E_{12}(i) & E_{13}(i) & E_{14}(i) \\
E_{12}(i)^{T} & E_{22}(i, j) & E_{23}(i) & E_{24}(i) \\
E_{13}(i)^{T} & E_{23}(i)^{T} & -\gamma I & 0 \\
E_{14}(i)^{T} & E_{24}(i)^{T} & 0 & -\gamma I
\end{array}\right], \\
& E_{11}(i, j):=\dot{X}\left(\alpha_{d}^{j}\right)+A\left(\alpha_{g}^{i}\right)^{T} X\left(\alpha_{g}^{i}\right)+X\left(\alpha_{g}^{i}\right) A\left(\alpha_{g}^{i}\right) \\
& +\hat{B}\left(\alpha_{g}^{i}\right) C_{2}\left(\alpha_{g}^{i}\right)+C_{2}\left(\alpha_{g}^{i}\right)^{T} \hat{B}\left(\alpha_{g}^{i}\right)^{T}, \\
& E_{12}(i):=\hat{A}\left(\alpha_{g}^{i}\right)+A\left(\alpha_{g}^{i}\right)^{T} \text {, } \\
& E_{13}(i):=X\left(\alpha_{g}^{i}\right) B_{1}\left(\alpha_{g}^{i}\right)+\hat{B}\left(\alpha_{g}^{i}\right) D_{21}\left(\alpha_{g}^{i}\right), \\
& E_{14}(i):=C_{1}\left(\alpha_{g}^{i}\right)^{T} \text {, } \\
& E_{22}(i, j):=-\dot{Y}\left(\alpha_{d}^{j}\right)+A\left(\alpha_{g}^{i}\right) Y\left(\alpha_{g}^{i}\right)+Y\left(\alpha_{g}^{i}\right) A\left(\alpha_{g}^{i}\right)^{T} \\
& +B_{2}\left(\alpha_{g}^{i}\right) \hat{C}\left(\alpha_{g}^{i}\right)+\hat{C}\left(\alpha_{g}^{i}\right)^{T} B_{2}\left(\alpha_{g}^{i}\right)^{T}, \\
& E_{23}(i):=B_{1}\left(\alpha_{g}^{i}\right) \text {, } \\
& E_{24}(i):=C_{1}\left(\alpha_{g}^{i}\right) Y\left(\alpha_{g}^{i}\right)+D_{12}\left(\alpha_{g}^{i}\right) \hat{C}\left(\alpha_{g}^{i}\right), \\
& {\left[\begin{array}{cc}
X\left(\alpha_{g}^{i}\right) & I \\
I & Y\left(\alpha_{g}^{i}\right)
\end{array}\right]>0}
\end{aligned}
$$

ここで, $\dot{X}\left(\alpha_{d}^{j}\right) \in \mathscr{S}^{n_{x}}, \dot{Y}\left(\alpha_{d}^{j}\right) \in \mathscr{S}^{n_{x}}\left(j=1, \ldots, n_{d}\right)$ は, 式 (8), (9) のパラメトリゼーションの下で $X(\alpha((t))$ お よび $Y(\alpha(t))$ を $t$ で偏微分することによって, 簡単に 求めることができる.

Step 4: 式 (2) のゲインスケジュールドコントローラ $K$ の係数行列を次式によって求める.

$$
\begin{aligned}
& A_{K}:=N^{-1}\left(X \dot{Y}+\hat{A}-X A Y-\hat{B} C_{2} Y-X B_{2} \hat{C}\right), \\
& B_{K}:=N^{-1} \hat{B}, C_{K}:=\hat{C}, N:=I-X Y
\end{aligned}
$$

本設計手法では，グリッディングを行った時点で閉 ループ系 $G$ の $\mathscr{L}_{2}$ ゲイン制約（式(7)）はグリッドされ た点上 $\left(\alpha(t) \in \mathscr{A}^{g}\right)$ のみで成立し, すべての $\alpha(t) \in \mathscr{A}$ で満足される保証はなくなることに注意する. さらに 式 (8) (12) のパラメトリゼーションにおいても, 関 数 $\bar{p}_{i}(\alpha(t))\left(i=1, \ldots, n_{p}\right)$ の選択法に決定的な指針は ない1. よって, 式(13) および(14)の LMI 条件は, $G$ に対してすべての $\alpha(t) \in \mathscr{A}, \dot{\alpha}(t) \in \mathscr{D}_{\alpha}$ において式 (7) の $\mathscr{L}_{2}$ ゲイン制約が成立する必要条件である. 文献 ${ }^{(5)}$ では, 式 (7)の $\mathscr{L}_{2}$ ゲイン制約を満足するゲインスケ ジュールドコントローラ設計のための必要十分条件が 無限次元の LMI 条件で与えられている. これまでこ のような無限次元 LMI を近似なしに解く手法は知ら れておらず, グリッディングや式 (8)〜(12) のパラメ トリゼーションは, 文献 ${ }^{(5)}$ の無限次元の LMI 条件を現 在入手可能な LMI ソルバ(8) で解くことのできる有限 次元の LMI 条件で近似するためのものである. 一般 化プラント $P$ の状態方程式 (1) の係数行列を $\alpha(t)$ の 線形関数に限定しない場合は, 今のところこの設計手 法が最も実用的である. また, グリッドを十分細かく 取ることによって, 実用的に十分高い性能が得られる ことが多い(6) (7).

3.2 統合化設計アルゴリズム本論文では, 前 節のゲインスケジュールドコントローラ $K$ の設計法に 基づいて, Hiramoto $ら^{(9)}$ による反復 LMI 近似を用い た統合化設計アルゴリズムを提案する. 本手法は, 文 献 ${ }^{(9)}$ ではLTI システムに対して適用され，その有効性 が示されているが, 本論文のようなゲインスケジュー ルド制御を考えた場合でも同様に適用可能である．以 下にその設計アルゴリズムを示す．本手法は，多目的 制御系設計などの BMI 問題をホモトピー法によって 解く際用いられているもの ${ }^{(10)}$ を元にしている.

Step 1: $k$ を繰り返し回数とする. $k=1$ とおく. 正数 $\varepsilon_{c}$ を決定する.

Step 2: 構造系の設計変数 $\beta^{k} \in \mathscr{B}$ を決定し, 式 (1)に 代入し，一般化プラント $P^{k}$ を求める. 適当なグリッ ディングを行い ${ }^{2}$, 前節の手法を用いて閉ループ系の $\mathscr{L}_{2}$ ゲインを最小化するゲインスケジュールドコント ローラ $K^{k}$ を求め, 達成された $\mathscr{L}_{2}$ ゲインを $\gamma^{k}$ とお $<. k \geq 2$ の場合, $\left|\gamma^{k}-\gamma^{k-1}\right|<\varepsilon_{c}$ ならば $K_{o p t}:=K^{k}$, $\beta_{o p t}:=\beta^{k}$ として設計終了. そのとき達成される閉ルー プ系の $\mathscr{L}_{2}$ ゲインは $\gamma^{k}$ となる. $\left|\gamma^{k}-\gamma^{k-1}\right| \geq \varepsilon_{c}$ ならば

\footnotetext{
1変数行列を式 $(8) \sim(12)$ のように $\alpha(t)$ の関数とおくことによっ て, それらを定数行列とおいた場合と比較して必ず $\gamma$ の意味で優れ た（少なくとも同等な）ゲインスケジュールドコントローラが求め られることは保証される。

2 本論文では, $k=1$ で決定したグリッティングをすべての $k$ で 変更しないと仮定する.
} 
Step 3 に行く.

Step 3: 行列 $\Delta X^{k} \in \mathscr{S}^{n_{x}}, \Delta Y^{k} \in \mathscr{S}^{n_{x}}, \Delta \hat{A}^{k} \in \mathscr{R}^{n_{x} \times n_{x}}$, $\Delta \hat{B}^{k} \in \mathscr{R}^{n_{x} \times n_{y}}$ および $\Delta \hat{C}^{k} \in \mathscr{R}^{n_{u} \times n_{x}}$ をそれぞれ式 (8)〜 (12) と同様のパラメトリゼーションを持つ（Step 2 で 得られた）行列 $X, Y, \hat{A}, \hat{B}$ および $\hat{C}$ の微小変動行列と する. 以下の行列不等式を $i=1, \cdots, n_{g}, j=1, \ldots, n_{d}$ に対して定義する.

$F^{k}(i, j)<0$,

$F^{k}(i, j):=\left[\begin{array}{cccc}F_{11}^{k}(i, j) & F_{12}^{k}(i) & F_{13}^{k}(i) & F_{14}^{k}(i) \\ F_{12}^{k}(i)^{T} & F_{22}^{k}(i, j) & F_{23}^{k}(i) & F_{24}^{k}(i) \\ F_{13}^{k}(i)^{T} & F_{23}^{k}(i)^{T} & -\left(\gamma+\Delta \gamma^{k}\right) I & 0 \\ F_{14}^{k}(i)^{T} & F_{24}^{k}(i)^{T} & 0 & -\left(\gamma+\Delta \gamma^{k}\right) I\end{array}\right]$, $F_{11}^{k}(i, j):=\dot{X}\left(\alpha_{d}^{j}\right)+\Delta \dot{X}^{k}\left(\alpha_{d}^{j}\right)$

$+\left(A\left(\alpha_{g}^{i}\right)+\Delta A^{k}\left(\alpha_{g}^{i}\right)\right)^{T}\left(X\left(\alpha_{g}^{i}\right)+\Delta X^{k}\left(\alpha_{g}^{i}\right)\right)$

$+\left(X\left(\alpha_{g}^{i}\right)+\Delta X^{k}\left(\alpha_{g}^{i}\right)\right)\left(A\left(\alpha_{g}^{i}\right)+\Delta A^{k}\left(\alpha_{g}^{i}\right)\right)$

$+\left(\hat{B}\left(\alpha_{g}^{i}\right)+\Delta \hat{B}_{K}^{k}\left(\alpha_{g}^{i}\right)\right)\left(C_{2}\left(\alpha_{g}^{i}\right)+\Delta C_{2}^{k}\left(\alpha_{g}^{i}\right)\right)$

$+\left(C_{2}\left(\alpha_{g}^{i}\right)+\Delta C_{2}^{k}\left(\alpha_{g}^{i}\right)\right)^{T}\left(\hat{B}\left(\alpha_{g}^{i}\right)+\Delta \hat{B}^{k}\left(\alpha_{g}^{i}\right)\right)^{T}$,

$F_{12}^{k}(i):=\hat{A}\left(\alpha_{g}^{i}\right)+\Delta \hat{A}^{k}\left(\alpha_{g}^{i}\right)+\left(A\left(\alpha_{g}^{i}\right)+\Delta A^{k}\left(\alpha_{g}^{i}\right)\right)^{T}$,

$F_{13}^{k}(i):=\left(X\left(\alpha_{g}^{i}\right)+\Delta X^{k}\left(\alpha_{g}^{i}\right)\right)\left(B_{1}\left(\alpha_{g}^{i}\right)+\Delta B_{1}^{k}\left(\alpha_{g}^{i}\right)\right)$

$+\left(\hat{B}\left(\alpha_{g}^{i}\right)+\Delta \hat{B}^{k}\left(\alpha_{g}^{i}\right)\right)\left(D_{21}\left(\alpha_{g}^{i}\right)+\Delta D_{21}^{k}\left(\alpha_{g}^{i}\right)\right)$,

$F_{14}^{k}(i):=\left(C_{1}\left(\alpha_{g}^{i}\right)+\Delta C_{1}^{k}\left(\alpha_{g}^{i}\right)\right)^{T}$,

$F_{22}^{k}(i, j):=-\left(\dot{Y}\left(\alpha_{d}^{j}\right)+\Delta \dot{Y}^{k}\left(\alpha_{d}^{j}\right)\right)$

$+\left(A\left(\alpha_{g}^{i}\right)+\Delta A^{k}\left(\alpha_{g}^{i}\right)\right)\left(Y\left(\alpha_{g}^{i}\right)+\Delta Y^{k}\left(\alpha_{g}^{i}\right)\right)$

$+\left(Y\left(\alpha_{g}^{i}\right)+\Delta Y^{k}\left(\alpha_{g}^{i}\right)\right)\left(A\left(\alpha_{g}^{i}\right)+\Delta A^{k}\left(\alpha_{g}^{i}\right)\right)^{T}$

$+\left(B_{2}\left(\alpha_{g}^{i}\right)+\Delta B_{2}^{k}\left(\alpha_{g}^{i}\right)\right)\left(\hat{C}\left(\alpha_{g}^{i}\right)+\Delta \hat{C}^{k}\left(\alpha_{g}^{i}\right)\right)$

$+\left(\hat{C}\left(\alpha_{g}^{i}\right)+\Delta \hat{C}^{k}\left(\alpha_{g}^{i}\right)\right)^{T}\left(B_{2}\left(\alpha_{g}^{i}\right)+\Delta B_{2}^{k}\left(\alpha_{g}^{i}\right)\right)^{T}$,

$F_{23}^{k}(i):=B_{1}\left(\alpha_{g}^{i}\right)+\Delta B_{1}^{k}\left(\alpha_{g}^{i}\right)$,

$F_{24}^{k}(i):=\left(C_{1}\left(\alpha_{g}^{i}\right)+\Delta C_{1}^{k}\left(\alpha_{g}^{i}\right)\right)\left(Y\left(\alpha_{g}^{i}\right)+\Delta Y^{k}\left(\alpha_{g}^{i}\right)\right)$

$+\left(D_{12}\left(\alpha_{g}^{i}\right)+\Delta D_{12}^{k}\left(\alpha_{g}^{i}\right)\right)\left(\hat{C}\left(\alpha_{g}^{i}\right)+\Delta \hat{C}^{k}\left(\alpha_{g}^{i}\right)\right)$,

$\left[\begin{array}{cc}X\left(\alpha_{g}^{i}\right)+\Delta X^{k}\left(\alpha_{g}^{i}\right) & I \\ I & Y\left(\alpha_{g}^{i}\right)+\Delta Y^{k}\left(\alpha_{g}^{i}\right)\end{array}\right]>0$

ここで, 行列 $\Delta A^{k}\left(\alpha_{g}^{i}\right) \in \mathscr{R}^{n_{x} \times n_{x}}, \Delta B_{1}^{k}\left(\alpha_{g}^{i}\right) \in \mathscr{R}^{n_{x} \times n_{w}}$, $\Delta B_{2}^{k}\left(\alpha_{g}^{i}\right) \in \mathscr{R}^{n_{x} \times n_{u}}, \Delta C_{1}^{k}\left(\alpha_{g}^{i}\right) \in \mathscr{R}^{n_{z} \times n_{x}}, \Delta C_{2}^{k}\left(\alpha_{g}^{i}\right) \in$ $\mathscr{R}^{n_{y} \times n_{x}}, \Delta D_{12}^{k}\left(\alpha_{g}^{i}\right) \in \mathscr{R}^{n_{z} \times n_{u}}$ および $\Delta D_{21}^{k}\left(\alpha_{g}^{i}\right) \in \mathscr{R}^{n_{y} \times n_{z}}$ は, 制御対象中の設計変数ベクトル $\beta^{k}$ が, 微小変動 $\Delta \beta^{k}:=\left[\Delta \beta_{1}^{k}, \ldots, \Delta \beta_{n_{\beta}}^{k}\right]^{T} \in \mathscr{R}^{n_{\beta}}$ によって $\beta^{k}+\Delta \beta^{k} \in \mathscr{B}$ と変動することによる一般化プラント $P$ の状態方程式 の係数行列 $A, B_{1}, B_{2}, C_{1}, C_{2}, D_{12}, D_{21}$ の変動の一次近 似である ${ }^{3}$.これらの係数行列の変動は, 係数行列が 設計変数べクトル $\beta$ の一般的な非線形関数で与えられ

${ }^{3}$ 式 (15) および (16) は, 設計変数べクトル $\beta \in \mathscr{R}^{n_{\beta}}$ の関数にも なっていることに注意する。
る場合は以下のような近似式で表すことができる.

$$
\begin{aligned}
\star^{k}\left(\beta^{k}+\Delta \beta^{k}\right) & \simeq \star^{k}\left(\beta^{k}\right)+\sum_{i=1}^{n_{\beta}} \Delta \beta_{i}^{k} \frac{\partial \star^{k}\left(\beta^{k}\right)}{\partial \beta_{i}^{k}} \\
& =\star^{k}\left(\beta^{k}\right)+\Delta \star^{k}\left(\beta^{k}, \Delta \beta^{k}\right)
\end{aligned}
$$

ここで, $\star^{k}\left(\beta^{k}\right)$ は, $P$ の状態方程式の係数行列を表し， $\partial \star^{k}\left(\beta^{k}\right) / \partial \beta_{i}^{k}$ は, その係数行列を $\beta^{k}$ の成分 $\beta_{i}^{k}$ で偏 微分した行列である. $\Delta \beta_{i}^{k}$ が十分小さい場合, 近似式 (17) は実際の変動量に対して十分高い精度を持つと考 えることができる. なお, 式(17)は, 係数行列が設計 変数 $\beta_{i}^{k}$ の線形関数である場合は近似なく厳密に成立 する.

Step 4: 式 (15) は, $\Delta$ つき変数に関する BMI となる. 本論文では変動が微小であると考え, 式 (15) の $\Delta$ に 関する二次項を無視した以下の近似条件を考える.

$$
\begin{aligned}
& \overline{F^{k}}(i, j)<0, \\
& \overline{F^{k}}(i, j):=\left[\begin{array}{cccc}
\overline{F_{11}^{k}}(i, j) & F_{12}^{k}(i) & \overline{F_{13}^{k}}(i) & F_{14}^{k}(i) \\
F_{12}^{k}(i)^{T} & F_{22}^{k}(i, j) & F_{23}^{k}(i) & F_{24}^{k}(i) \\
F_{13}^{k}(i)^{T} & F_{23}^{k}(i)^{T} & -\left(\gamma+\Delta \gamma^{k}\right) I & 0 \\
F_{14}^{k}(i)^{T} & F_{24}^{k}(i)^{T} & 0 & -\left(\gamma+\Delta \gamma^{k}\right) I
\end{array}\right], \\
& \overline{F_{11}^{k}}(i, j):=\dot{X}\left(\alpha_{d}^{j}\right)+\Delta \dot{X}^{k}\left(\alpha_{d}^{j}\right) \\
& +A\left(\alpha_{g}^{i}\right)^{T} X\left(\alpha_{g}^{i}\right)+X\left(\alpha_{g}^{i}\right) A\left(\alpha_{g}^{i}\right) \\
& +A\left(\alpha_{g}^{i}\right)^{T} \Delta X^{k}\left(\alpha_{g}^{i}\right)+\Delta X^{k}\left(\alpha_{g}^{i}\right) A\left(\alpha_{g}^{i}\right) \\
& +\Delta A^{k}\left(\alpha_{g}^{i}\right)^{T} X\left(\alpha_{g}^{i}\right)+X\left(\alpha_{g}^{i}\right) \Delta A^{k}\left(\alpha_{g}^{i}\right) \\
& +\hat{B}\left(\alpha_{g}^{i}\right) C_{2}\left(\alpha_{g}^{i}\right)+C_{2}\left(\alpha_{g}^{i}\right)^{T} \hat{B}\left(\alpha_{g}^{i}\right)^{T} \\
& +\hat{B}\left(\alpha_{g}^{i}\right) \Delta C_{2}^{k}\left(\alpha_{g}^{i}\right)+\Delta C_{2}^{k}\left(\alpha_{g}^{i}\right)^{T} \hat{B}\left(\alpha_{g}^{i}\right)^{T} \\
& +\Delta \hat{B}_{K}^{k}\left(\alpha_{g}^{i}\right) C_{2}\left(\alpha_{g}^{i}\right)+C_{2}\left(\alpha_{g}^{i}\right)^{T} \Delta \hat{B}_{K}^{k}\left(\alpha_{g}^{i}\right)^{T}, \\
& \overline{F_{13}^{k}}(i):=X\left(\alpha_{g}^{i}\right) B_{1}\left(\alpha_{g}^{i}\right)+X\left(\alpha_{g}^{i}\right) \Delta B_{1}^{k}\left(\alpha_{g}^{i}\right) \Delta X^{k}\left(\alpha_{g}^{i}\right) B_{1}\left(\alpha_{g}^{i}\right) \\
& +\hat{B}\left(\alpha_{g}^{i}\right) D_{21}\left(\alpha_{g}^{i}\right)+\hat{B}\left(\alpha_{g}^{i}\right) \Delta D_{21}^{k}\left(\alpha_{g}^{i}\right)+\Delta \hat{B}^{k}\left(\alpha_{g}^{i}\right) D_{21}\left(\alpha_{g}^{i}\right), \\
& \overline{F_{22}^{k}}(i, j):=-\left(\dot{Y}\left(\alpha_{d}^{j}\right)+\Delta \dot{Y}^{k}\left(\alpha_{d}^{j}\right)\right) \\
& +A\left(\alpha_{g}^{i}\right) Y\left(\alpha_{g}^{i}\right)+Y\left(\alpha_{g}^{i}\right) A\left(\alpha_{g}^{i}\right)^{T} \\
& +A\left(\alpha_{g}^{i}\right) \Delta Y^{k}\left(\alpha_{g}^{i}\right)+\Delta Y^{k}\left(\alpha_{g}^{i}\right) A\left(\alpha_{g}^{i}\right)^{T} \\
& +\Delta A^{k}\left(\alpha_{g}^{i}\right) Y\left(\alpha_{g}^{i}\right)+Y\left(\alpha_{g}^{i}\right) \Delta A^{k}\left(\alpha_{g}^{i}\right)^{T} \\
& +B_{2}\left(\alpha_{g}^{i}\right) \hat{C}\left(\alpha_{g}^{i}\right)+\hat{C}\left(\alpha_{g}^{i}\right)^{T} B_{2}\left(\alpha_{g}^{i}\right)^{T} \\
& +B_{2}\left(\alpha_{g}^{i}\right) \Delta \hat{C}^{k}\left(\alpha_{g}^{i}\right)+\Delta \hat{C}^{k}\left(\alpha_{g}^{i}\right)^{T} B_{2}\left(\alpha_{g}^{i}\right)^{T} \\
& +\Delta B_{2}^{k}\left(\alpha_{g}^{i}\right) \hat{C}\left(\alpha_{g}^{i}\right)+\hat{C}\left(\alpha_{g}^{i}\right)^{T} \Delta B_{2}^{k}\left(\alpha_{g}^{i}\right)^{T}, \\
& \overline{F_{24}^{k}}(i):=C_{1}\left(\alpha_{g}^{i}\right) Y\left(\alpha_{g}^{i}\right)+C_{1}\left(\alpha_{g}^{i}\right) \Delta Y^{k}\left(\alpha_{g}^{i}\right)+\Delta C_{1}^{k}\left(\alpha_{g}^{i}\right) Y\left(\alpha_{g}^{i}\right) \\
& +D_{12}\left(\alpha_{g}^{i}\right) \hat{C}\left(\alpha_{g}^{i}\right)+D_{12}\left(\alpha_{g}^{i}\right) \Delta \hat{C}^{k}\left(\alpha_{g}^{i}\right)+\Delta D_{12}^{k}\left(\alpha_{g}^{i}\right) \hat{C}\left(\alpha_{g}^{i}\right) \\
& i=1, \ldots, n_{g}, j=1, \ldots, n_{d}
\end{aligned}
$$

式 (18) は，すべての変動行列（ $\Delta$ つき行列）に関する LMI である. さらに, すべての変動行列が “小さい” 
ことを, 次式のように $\Delta \beta^{k}$ および, 変動行列変数の最 大特異值で制限する.

$$
\sigma_{\max }(\Delta \bullet) \leq \kappa \sigma_{\max }(\bullet), 0<\kappa<1
$$

ここで,・には変動を仮定する行列変数の記号 $\left(\beta^{k}, \hat{X}\right.$, $\hat{A}$ 等) が入る. 式 (19) と等価な LMI 制約は, 次式で 与えられる.

$$
\left[\begin{array}{cc}
\kappa \sigma_{\max }(\bullet) I & \Delta \bullet \\
(\Delta \bullet)^{T} & \kappa \sigma_{\max }(\bullet) I
\end{array}\right] \geq 0
$$

式(18), (16), $\beta^{k}$ の上下限に関する線形制約 $\underline{\beta}<\beta^{k} \leq \bar{\beta}$ （式(5)）および式(20)はいずれも調整される変数に対 する LMI である.これらと $\Delta \gamma^{k} \leq 0$ の線形制約を連 立させ, $\Delta \gamma^{k}$ を最小化する変数を求める. 解がない場 合は, 探索領域を現在の設計変数のより近傍付近に制 限するように，

$$
\kappa:=\eta \kappa, 0<\eta<1
$$

と更新し, 再度上記連立 LMI を解く. 制約 $\Delta \gamma^{k} \leq 0$ $\left(\gamma^{k+1} \leq \gamma^{k}\right)$ を満たす解が得られるまで式 (21) の更新 を絽り返して ( $\kappa$ を小さくして), 上記連立 LMI を解く. 解が得られた時点で, $\Delta \beta^{k}$ を用いて, $\beta^{k+1}:=\beta^{k}+\Delta \beta^{k}$, $k \leftarrow k+1$ と更新して, Step 2 に戻る.

提案する設計アルゴリズムの特徵を以下に述べる.

a. 著者の知る限り, 従来の統合化設計アルゴリズム はすべて LTI システムを対象としたものであった. 本アルゴリズムは, グリッディングによる近似を経 てはいるものの LPV システムに対してはじめて提 案された統合化設計手法である.

b. Step 3 において， $\Delta \gamma<0$ が成立するまで変数の更 新量を支配する定数 $\kappa$ を調整している.これより, 同一グリッディングの下で初期設計パラメータ $\beta$ の局所的最適解への収束は保証される.

c. 式(15)の BMIの $\Delta$ に関する 2 次項を無視した LMI （式 (18)）が意味を持つように, $\beta$ の更新量を小さ くしていることが, 結果として式 (17) の一次近似 の精度も保っている. よって, 本アルゴリズムは, $P$ の状態方程式中の係数行列が $\beta$ の非線形関数と なっていても適用可能である. 文献 ${ }^{(11)(12)}$ では, LTI システムに対し統合化設計問題を BMI 問題で定式 化し, 構造系およびコントローラの設計変数の一方 を固定した LMI を絽り返し解く手法が提案されて いるが, これらの手法には, 制御対象の状態方程式 （またはディスクリプタ表現）の係数行列が制御対 象に存在する設計変数の線形関数であるという制 限があった. その意味で, 本アルゴリズムはこれら の文献 ${ }^{(11)(12)}$ の手法を超えている.
注意 $1 P$ の状態方程式の係数行列のうち, 行列 $A, B_{1}$, $C_{1}$ が $\alpha(t)$ の線形関数で, 他の行列が定数行列の場合, ゲインスケジュールドコントローラの設計は（グリッ ディングなどの近似なく）著しく簡単化される(4).す なわち, 集合 $\mathscr{A}$ を $n_{\alpha}$ 次元の超直方体之考えた場合 の端点 $\mathscr{V}(\mathscr{A}):=\left\{\alpha_{v}^{1}, \ldots, \alpha_{v}^{n_{\nu}}\right\}, n_{v}:=2^{n_{\alpha}}$ 上で, 以下 の $L M I$ 中の $\gamma>0$ を最小化する定数行列 $X, Y \in \mathscr{S}^{n_{x}}$, $\hat{A} \in \mathscr{R}^{n_{x} \times n_{x}}, \hat{B} \in \mathscr{R}^{n_{x} \times n_{y}}, \hat{C} \in \mathscr{R}^{n_{u} \times n_{x}} \quad\left(i=1, \ldots, n_{v}\right)$ を 求めればよい4.

$$
\begin{aligned}
& H(i)<0, \\
& H(i):=\left[\begin{array}{cccc}
H_{11}(i) & H_{12}(i) & H_{13}(i) & H_{14}(i) \\
H_{12}(i)^{T} & H_{22}(i) & H_{23}(i) & H_{24}(i) \\
H_{13}(i)^{T} & H_{23}(i)^{T} & -\gamma I & 0 \\
H_{14}(i)^{T} & H_{24}(i)^{T} & 0 & -\gamma I
\end{array}\right], \\
& H_{11}(i):=A\left(\alpha_{v}^{i}\right)^{T} X+X A\left(\alpha_{v}^{i}\right)+\hat{B} C_{2}+C_{2}^{T} \hat{B}, \\
& H_{12}(i):=\hat{A}+A\left(\alpha_{v}^{i}\right)^{T}, H_{13}(i):=X B_{1}\left(\alpha_{v}^{i}\right)+\hat{B} D_{21} \text {, } \\
& H_{14}(i):=C_{1}\left(\alpha_{v}^{i}\right) \text {, } \\
& H_{22}(i):=A\left(\alpha_{v}^{i}\right) Y+Y A\left(\alpha_{v}^{i}\right)^{T}+B_{2} \hat{C}+\hat{C}^{T} B_{2}, \\
& H_{23}(i):=B_{1}\left(\alpha_{v}^{i}\right), H_{24}(i):=C_{1}\left(\alpha_{v}^{i}\right) Y+D_{12} \hat{C}, \\
& {\left[\begin{array}{ll}
X & I \\
I & Y
\end{array}\right] \geq 0}
\end{aligned}
$$

本論文で提案した統合化設計アルゴリズムは, $P$ に関し て文献 ${ }^{(4)}$ の条件が成立している場合でも, 式(13), (14) で与えられているゲインスケジュールドコントローラ 設計条件を式 (22), (23) に変更することにより, その まま用いることができる.

注意 2 本論文では, 最小化する評価指標を閉ループ系 の $\mathscr{L}_{2}$ ゲイン $\gamma$ とした. 構造の強度や重量等の構造系 に関する $\left(\beta \in \mathscr{B}\right.$ のみに依存する）評価指標を $J_{s}$ と おき $J_{s}$ の最小化問題が $L M I$ に帰着されるか近似可能 な場合は, 以下の新しい重みつき和で表される評価指 標 $J:=\lambda \gamma+(1-\lambda) J_{s}, 0 \leq \lambda \leq 1$ を定義すれば, $J$ を 最小化するために本論文で提案した設計アルゴリズム をそのまま使F できる.

注意 3 本論文では, スケジューリングパラメータ $\alpha(t)$ と構造系の設計変数 $\beta$ は別個のものであると仮定し， 問題設定および設計アルゴリズムの提案を行ってきた. もし $\alpha(t)$ の一部または全部が, 式 (3)や(4)の下で変 動はするものの設計者によって調整可能な場合は, そ れらの調整可能なスケジューリングパラメータを何ら かの指標に基づいて適切に設計することが可能となり， 新たな設計自由度が発生する.このような問題の例と

${ }^{4}$ 文献 $^{(4)}$ では, コントローラ設計のためのより規模の小さい LMI 条件が導出されている. 
して, 外界状況の変化やタスクの切り替えに応じて形 状などを変化させ, 特性を適切に変更できる適応構造 物の設計問題を挙げることができる. 本論文で提案し た反復 $L M I$ 近似に基づく設計手法は, $\alpha(t)$ の一部ま たは全部が調整可能な場合でも, 閉ループ系の $\mathscr{L}_{2}$ ゲ インを評価とし, 集合 $\mathscr{A}$ および $\mathscr{D}_{\alpha}\left(\right.$ 集合 $\mathscr{A}^{8}$ および $\left.\mathscr{D}_{\alpha}^{g}\right)$ が, $\alpha(t)$ の調整下でも不変な場合は, 調整可能 な $\alpha(t)$ を $\beta$ に含ませることによって, 統合化設計を 行うことができる. $\alpha(t)$ を自由に設計できるこのよう な問題は, 非常に興味深く, 今後より詳細に検討する 予定である.

\section{4. 設 計 例}

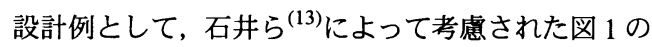
モデルで表される回転型倒立振子を考える. 図 1 にお いて, $q_{0}(t), q_{1}(t)$ および $u(t)$ は，それぞれ台軸角度， 振子角度および台軸への入カトルクであり, $m_{0}, m_{1}, l_{0}$, $l_{1}, J_{0}$ および $J_{1}$ は, それぞれ台軸質量, 振子質量, 台 軸長さ, 振子長さ, 台軸重心まわりの慣性モーメント および振子重心まわりの慣性モーメントである. $d_{0}$ お よび $d_{1}$ をそれぞれ台軸の回転関節および台軸と振子 間の回転関節での粘性摩擦係数とする. 状態べクトル $x(t):=\left[q_{0}(t) q_{1}(t) \dot{q}_{0}(t) \dot{q}_{1}(t)\right]^{T}$ とすると, 本システム の状態方程式は, 以下のように表される ${ }^{(13)}$.

$\dot{x}(t)=R(x(t), u(t))$,

$$
\begin{aligned}
& R(x(t), u(t))=\left[\begin{array}{c}
\dot{q}_{0}(t) \\
\dot{q}_{1}(t) \\
r_{1}(x(t))+s_{1}(x(t)) u(t) \\
r_{2}(x(t))+s_{2}(x(t)) u(t)
\end{array}\right] \\
& r_{1}(x)=-\frac{1}{\operatorname{det} M(x)}\left\{2 v_{2}\left(v_{2}+v_{4}\right) \sin q_{1} \cos q_{1} \cdot \dot{q}_{0} \dot{q}_{1}\right. \\
& +d_{0}\left(v_{2}+v_{4}\right) \dot{q}_{0}-v_{3}\left(v_{2}+v_{4}\right) \sin q_{1} \cdot \dot{q}_{1}^{2} \\
& +v_{2} v_{3} \sin q_{1} \cos ^{2} q_{1} \cdot \dot{q}_{0}^{2}-v_{3} d_{1} \cos q_{1} \cdot q_{1}^{2} \\
& \left.+v_{3} v_{5} \sin q_{1} \cos q_{1}\right\},
\end{aligned}
$$$$
r_{2}(x)=-\frac{1}{\operatorname{det} M(x)}\left\{-2 v_{2} v_{3} \sin q_{1} \cos ^{2} q_{1} \cdot \dot{q}_{0} \dot{q}_{1}\right.
$$$$
-v_{3} d_{0} \cos q_{1} \cdot \dot{q}_{0}+v_{3}^{2} \sin q_{1} \cos q_{1} \cdot \dot{q}_{1}^{2}
$$$$
-v_{2}\left(v_{1}+v_{2} \sin ^{2} q_{1}\right) \sin q_{1} \cos q_{1} \cdot \dot{q}_{0}^{2}
$$$$
\left.+d_{1}\left(v_{1}+v_{2} \sin ^{2} q_{1}\right) \dot{q}_{1}-v_{5}\left(v_{1}+v_{2} \sin ^{2} q_{1}\right) \sin q_{1}\right\},
$$$$
s_{1}(x)=\frac{1}{\operatorname{det} M(x)}\left(v_{2}+v_{4}\right), s_{2}=-\frac{1}{\operatorname{det} M(x)} v_{3} \cos q_{1} \text {, }
$$$$
v_{1}=J_{0}+\frac{m_{0} l_{0}^{2}}{4}+m_{1} l_{0}^{2}, v_{2}=\frac{m_{1} l_{1}^{2}}{4}, v_{3}=\frac{m_{1} l_{0} l_{1}}{2},
$$$$
v_{4}=J_{1}, v_{5}=\frac{m_{1} g l_{1}}{2}, M(x)=\left[\begin{array}{cc}
v_{1}+v_{2} \sin ^{2} q_{1} v_{3} \cos q_{1} \\
v_{3} \cos q_{1} & v_{2}+v_{4}
\end{array}\right]
$$

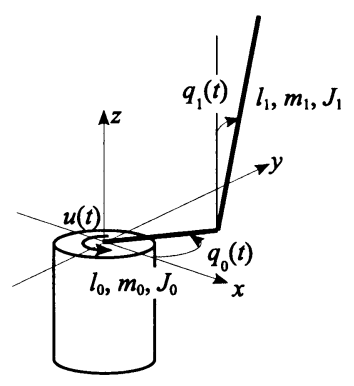

Fig. 1 Model of the Inverted Pendulum

Table 1 Physical Parameters

\begin{tabular}{cr}
\hline Physical Parameter & Value [Unit] \\
\hline$\rho$ & $2700\left[\mathrm{~kg} / \mathrm{m}^{3}\right]$ \\
$\phi_{0}$ & $0.05[\mathrm{~m}]$ \\
$\phi_{1}$ & $0.01[\mathrm{~m}]$ \\
$d_{0}$ & $0.1[\mathrm{Ns} / \mathrm{m}]$ \\
$d_{1}$ & $0.01[\mathrm{Ns} / \mathrm{m}]$ \\
\hline
\end{tabular}

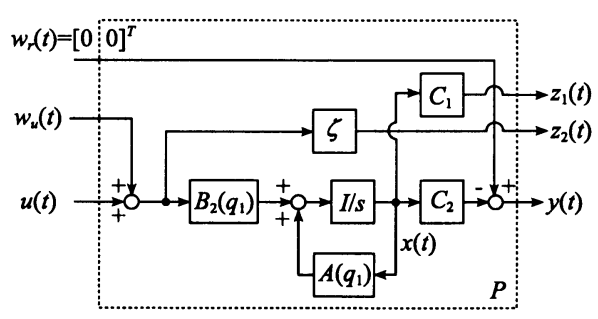

Fig. 2 Generalized Plant $P$

本設計例では, 台軸および振子は共に密度 $\rho$ の同一 材質で製作され，かつそれぞれ直径 $\phi_{0}, \phi_{1}$ の円断面を 持つという仮定の下で, 制御対象中の設計変数を台軸 および振子の長さ $l_{0}, l_{1}$ とし, $\beta:=\left[l_{0} l_{1}\right]^{T}$ とおく. 両 設計変数の変動に伴い, 台軸や振子の質量や慣性モ一 メントが変化し, システムの動特性が変化する. $\beta$ の 初期值を $\beta^{0}=\left[l_{0}^{0} l_{1}^{0}\right]^{T}, l_{0}^{0}=l_{1}^{0}=1.0[\mathrm{~m}]$ とし, 式 (5)の $\beta$ の上下限をそれぞれ $\bar{\beta}:=\left[\begin{array}{ll}10 & 10\end{array}\right]^{T}, \underline{\beta}:=\left[\begin{array}{ll}0.1 & 0.1\end{array}\right]^{T}$ と する. 他の物理パラメータ値を表 1 に示す.

式 (24) の LPV システム近似を求める. 式 (24) を不 安定平衡点 (倒立状態) $x_{e}:=\left[\begin{array}{llll}0 & 0 & 0 & 0\end{array}\right]^{T}$ まわりで線形 化を行う際, 振子角度 $q_{1}(t)$ に関する（三角関数から 構成される）非線形項はそのまま残し，スケジューリ ングパラメータ $\alpha(t)$ を $\alpha(t):=q_{1}(t)$ ととったものを式 (24) の LPV システム近似とする. 得られた LPV シス テムに対する一般化プラントのブロック線図を図 2 に 示す. ここで, $w_{r}(t)=[00]^{T}$ および $w_{u}(t)$ は台軸と振 子の目標值およびアクチュエータから混入する入力外 乱である. $z_{1}(t):=\left[q_{0}(t) q_{1}(t)\right]^{T}$ であり, $\zeta>0$ は, 操 
作量 $u(t)$ を $z_{2}(t):=\zeta u(t)$ で評価するためのスカラ重み である. $w(t):=\left[w_{r}(t)^{T} w_{u}(t)\right]^{T}, z(t):=\left[z_{1}(t)^{T} z_{2}(t)\right]^{T}$ および $y(t)=w_{r}(t)-\left[q_{0}(t) q_{1}(t)\right]^{T}$ とおくと, 式 $(1)$ の 一般化プラント $P$ の係数行列は以下のようになる.

$$
\begin{gathered}
A(\alpha(t), \beta)=\left[a_{i j}\right], B_{2}(\alpha(t), \beta)=\left[b_{i}\right], i, j=1, \ldots, 4, \\
B_{1}:=\left[0_{4 \times 2} B_{2}(\alpha(t), \beta)\right], C_{1}=\left[\begin{array}{ll}
I & 0_{2 \times 2}
\end{array}\right], C_{2}:=-C_{1}, \\
D_{11}:=0_{3 \times 3}, D_{12}:=\left[\begin{array}{lll}
0 & \zeta
\end{array}\right]^{T}, D_{21}:=\left[\begin{array}{ll}
I & 0_{2 \times 1}
\end{array}\right]
\end{gathered}
$$

$A(\alpha(t), \beta)$ および $B_{2}(\alpha(t), \beta)$ の詳細は付録で示す.

$\zeta=1$ として，3.2 節で提案された設計アルゴリズ ムによって $\beta_{o p t}$ およびゲインスケジュールドコント ローラ $K_{\text {opt }}$ を求めた. 式(8)-(12)の各行列は, それぞ れ $X=X_{0}+\alpha(t) X_{1}, Y=Y_{0}+\alpha(t) Y_{1}, \hat{A}=\hat{A}_{0}+\alpha(t) \hat{A}_{1}$, $\hat{B}=\hat{B}_{0}+\alpha(t) \hat{B}_{1}, \hat{C}=\hat{C}_{0}+\alpha(t) \hat{C}_{1}\left(X_{i}, Y_{i} \in \mathscr{S}^{4}, \hat{A}_{i} \in\right.$ $\left.\mathscr{R}^{4 \times 4}, \hat{B}_{i} \in \mathscr{R}^{4 \times 2}, \hat{C}_{i} \in \mathscr{R}^{1 \times 4}, i=0,1\right)$ とおき, スケジ ユーリングパラメータ $\alpha(t)$ は, $\mathscr{A}^{g}:=\left\{\alpha_{g}^{1}, \ldots, \alpha_{g}^{n_{g}}\right\}$, $\alpha_{g}^{i}=\pi[-1 / 6+(i-1) / 60] \quad(i=1, \ldots, 21)$ のようにグ リッディングした. 式 (4) の $\dot{\alpha}(t)$ に関する制約は, $\mathscr{D}_{\alpha}=\{\dot{\alpha}(t) \mid-4 \leq \dot{\alpha}(t) \leq 2\}$ とした. なお, 集合 $\mathscr{D}_{\alpha}$ は, 試行的な統合化設計後に $\dot{\alpha}(t)=\dot{q}_{1}(t)$ の初期值応 答を計算し決定した.

以上の条件下で, 提案する設計手法を用いて統合 化設計を行った結果を表 2 に示す. 計算には, PC (CPU: Intel Pentium IV 3.0GHz, RAM: 512MB) 上で MATLAB を用い約 30 分を要した. 初期時と比較して, 閉ループ系の $\mathscr{L}_{2}$ ゲインは約 $42 \%$ 隇少していることが 分かる. 図 3 に, $x(0)=\left[\begin{array}{llll}0 & \pi / 6 & 0 & 0\end{array}\right]^{T}$ としたときの初 期值応答を示す. なお, 以下で示されるすべての応答 は, 制御対象を式 (24)の非線形状態方程式とした非線 形シミュレーションの結果である. 初期時, 最適化後 共にゲインスケジュールド制御により安定化が達成さ れているが, 最適化後は, 初期時と比べて操作量 $u(t)$ の最大絶対值は小さくなっているにも関わらず, $q_{0}(t)$ および $q_{1}(t)$ の応答は明らかに改善しており, 統合化 設計の効果が現れている. なお, 本設計例においては, 異なる $l_{0}, l_{1}$ の初期值から最適化を行った場合も, 結 果は本章で示したものとほぼ同様であった.

ゲインスケジュールド制御の有効性を確認するため, 最適化された設計変数 $\beta_{o p t}$ で式 (24) を平衡点 $x_{e}$ まわ りに線形化した後 $q_{1}(t)$ を微小と仮定して求められた 倒立振子の LTI 近似システムに対して, 本設計例と同 一の $w, z(\zeta=1)$ で閉ループ系の $\mathscr{H}_{\infty}$ ノルムを最小化 するコントローラを設計し5, 図 3 と同じ初期条件で 非線形シミュレーションを行った結果を図 4 に示す.

${ }^{5} \mathrm{LTI}$ システムにおいては, $\mathscr{L}_{2}$ ゲインと $\mathscr{H}_{\infty}$ ノルムの定義は等 価である.
Table 2 Result of the Integrated Design

\begin{tabular}{rrr}
\hline & Initial Value & Optimized Value \\
\hline$\gamma$ & 110.1 & 63.7 \\
$l_{0}$ & $1.0[\mathrm{~m}]$ & $0.812[\mathrm{~m}]$ \\
$l_{1}$ & $1.0[\mathrm{~m}]$ & $0.418[\mathrm{~m}]$ \\
\hline
\end{tabular}
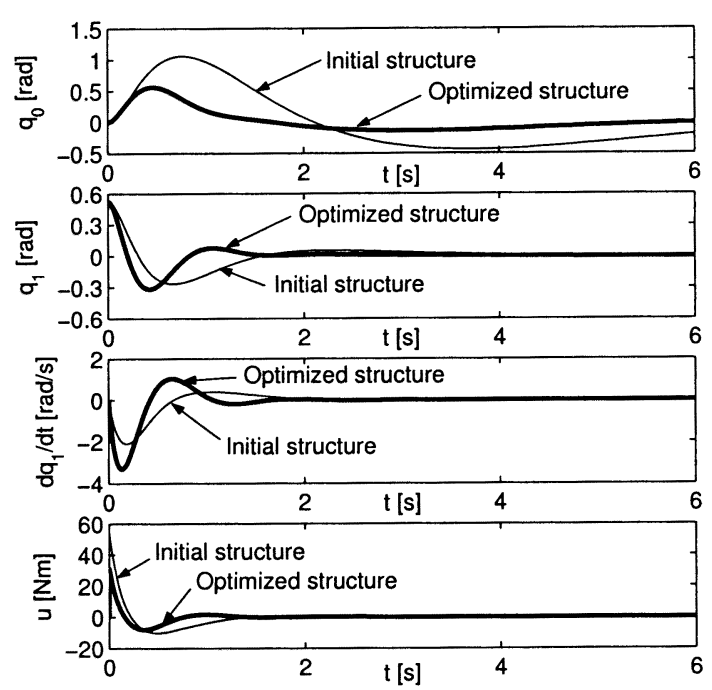

Fig. 3 Initial Response (Comparison between the Initial and the Optimized Structure)
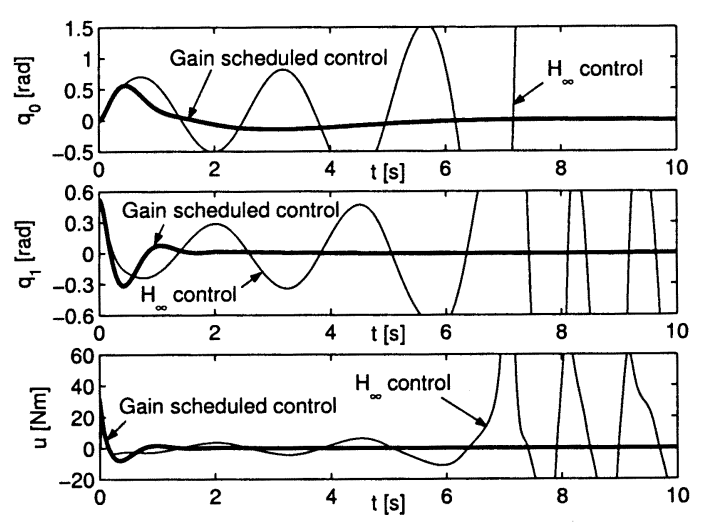

Fig. 4 Initial Response (Comparison between the Gain Scheduled Control and $\mathscr{H}_{\infty}$ Control Law)

ゲインスケジュールドコントローラでは, システムは 安定化されているのに対し, LTI コントローラである 舟寺ントローラでは, 閉ループ系を安定化すること が出来ず応答は発散している. 以上の結果から, 本論 文で提案された構造系とゲインスケジュールド制御系 の統合化設計の有効性が示された. 


\section{5. 結 言}

本論文では, LPV システムで表される制御対象中 に存在する設計変数と, ゲインスケジュールドコント ローラを同時に最適設計する一手法を提案した. 本論 文の内容を以下にまとめる.

・閉ループ系の $\mathscr{L}_{2}$ ゲインを規範として, LPV シス テムに対する構造系と制御系の統合化設計問題を 定式化した.

・ 反復 LMI 近似による繰り返し設計アルゴリズム を提案した. 本アルゴリズムは, 制御対象の状態 方程式の係数行列が構造系の設計変数の非線形関 数となっている場合でも, 設計変数 $\beta$ を局所的最 適解に収束させることができる.

・ 回転型倒立振子に対する設計例によって, 提案手 法の有効性を示した.

今後の課題を以下に示す.

・構造系の設計変数がスケジューリングパラメータ と一致する場合に対する検討.

・提案する設計手法の実システムへの適用

\section{文献}

(1) Onoda, J. and Haftka, R. T., An Approach to Structure/Control Simultaneous Optimization for Large Flexible Spacecraft, AIAA J., Vol. 25, No. 8 (1987), pp. 1133-1138.

(2) Obinata, G., Simultaneous Optimal Design of Structural and Control Systems, J. of the Society of Instrument and Control Engineers, Vol. 36, No. 4 (1997), pp. 254-261.

(3) Kajiwara, I., Simultaneous Design of Structure and Control System Considering Performance and Stability, Systems, Control and Information, Vol. 44, No. 11 (2000), pp. 623-631.

(4) Apkarian, P. et al., Self-Scheduled $H_{\infty}$ Control of Linear Parameter-varying Systems: a Design Example, Automatica, Vol. 31, No. 9 (1995), pp. 1251-1261.

(5) Apkarian, P. and Adams, R. J., Advanced GainScheduling Techniques for Uncertain Systems, IEEE Trans. on Control Systems Technology, Vol. 6, No. 1 (1998), pp. 21-32.

(6) Wu, F. and Grigoriadis, K. M., LPV Systems with Parameter-varying Time Delays: Analysis and Control, Automatica, Vol. 37, No. 2 (2001), pp. 221-229.

(7) Tuan, H. D. et al., Nonlinear $\mathscr{H}_{\infty}$ Control for an Integrated Suspension System via Parameterized Linear Matrix Inequality Characterization, IEEE Trans. on Control Systems Technology, Vol. 9, No. 1 (2001), pp. 175-185.

(8) Gahinet, P. et al., LMI Control Toolbox for Use with MATLAB, (1995), The MathWorks, Inc.

(9) Hiramoto, K. and Grigoriadis, K. M., Integrated Design of Structural and Control Systems with a Homotopy Like Iterative Method, Proc. of American Control Conference, (2005-6), pp. 2510-2515.
(10) Hassibi, A. et al., Low-Authority Controller Design via Convex Optimization, J. Guidance, Control, and Dynamics, Vol. 22, No. 6 (1999), pp. 862-872.

(11) Tanaka, H. and Sugie, T., General Framework and BMI Formulae for Simultaneous Design of Structure and Control Systems, Trans. of the Society of Instrument and Control Engineers, Vol. 34, No. 1 (1998), pp. 27-33.

(12) Lu, J. and Skelton, R. E., Integrating Structure and Control Design to Achieve Mixed $H_{2} / H_{\infty}$ Performance, Int. J. of Control, Vol. 73, No. 16 (2000), pp. 1449-1462.

(13) Ishii, C. et al., Swing-Up and Stabilization Control for an Inverted Pendulum System via Nonlinear Robust $H_{\infty}$ Control, Trans. of the Japan Society of Mechanical Engineers, Series C, Vol. 62, No. 599 (1996), pp. 26662673.

\section{付 録}

式 (25) の行列 $A(\alpha(t), \beta)=\left[a_{i j}\right], B_{2}(\alpha(t), \beta)=\left[b_{i}\right](i, j=$ $1, \ldots, 4)$ は, 以下のように表される.

$$
\begin{aligned}
& a_{11}=a_{12}=a_{14}=a_{21}=a_{22}=a_{23}=a_{31}=a_{41}=0, \\
& a_{13}=a_{24}=1, b_{1}=b_{2}=0, \\
& a_{32}=\frac{\xi_{32}}{\psi^{2}}, a_{33}=\frac{\xi_{33} l_{1}^{3}}{\psi}, a_{34}=\frac{\xi_{34}}{\psi}, a_{42}=\frac{\xi_{42}}{\psi^{2}}, \\
& a_{43}=\frac{\xi_{43} l_{0} l_{1}^{2}}{\psi}, a_{44}=\frac{\xi_{44}}{\psi}, b_{3}=\frac{\chi_{3} l_{1}^{3}}{\psi}, b_{4}=\frac{\chi_{4} l_{0} l_{1}^{2}}{\psi}, \\
& \psi=\psi_{1} l_{0}^{3} l_{1}^{3}+\psi_{2} l_{1}^{6}+\psi_{3} l_{0}^{2} l_{1}^{4}, \\
& \psi_{1}=\frac{1}{3}\left(\frac{1}{3} \theta_{0}+\theta_{1}\right) \theta_{1}, \psi_{2}=\frac{1}{12} \theta_{1}^{2} \sin ^{2} \alpha(t), \psi_{3}=-\frac{1}{4} \theta_{1}^{2} \cos ^{2} \alpha(t) \\
& \xi_{32}=\xi_{321} l_{0}^{4} l_{1}^{7}+\xi_{322} l_{0} l_{1}^{10}+\xi_{323} l_{0}^{3} l_{1}^{8}, \\
& \xi_{321}=-\frac{1}{12}\left(\frac{1}{3} \theta_{0}+\theta_{1}\right) \theta_{1}^{3} g \cos 2 \alpha(t), \\
& \xi_{322}=\frac{1}{48} \theta_{1}^{4} g \sin ^{2} \alpha(t), \xi_{323}=\frac{1}{16} \theta_{1}^{4} g \cos ^{2} \alpha(t)\left(4 \cos ^{2} \alpha(t)-1\right), \\
& \xi_{33}=-\frac{1}{3} d_{0} \theta_{1}, \xi_{34}=\frac{1}{2} d_{1} \theta_{1} \cos \alpha(t), \\
& \xi_{42}=\xi_{421} l_{0}^{3} l_{1}^{8}+\xi_{422} l_{1}^{1} 1+\xi_{423} l_{0}^{2} l_{1}^{9}+\xi_{424} l_{0}^{6} l_{1}^{5}+\xi_{425} l_{0}^{5} l_{1}^{6}, \\
& \xi_{421}=\frac{1}{12}\left(\frac{1}{3} \theta_{0}+\theta_{1}\right) \theta_{1}^{3} g \sin ^{2} \alpha(t) \cos \alpha(t), \\
& \xi_{422}=\frac{1}{96} \theta_{1}^{4} g \sin ^{4} \alpha(t) \cos \alpha(t), \\
& \xi_{423}=-\frac{1}{32} \theta_{1}^{4} g \sin ^{2} \alpha(t) \cos \alpha(t)\left(\cos ^{2} \alpha(t)+2\right), \\
& \xi_{424}=\frac{1}{6}\left(\frac{1}{3} \theta_{0}+\theta_{1}\right)^{2} \theta_{1}^{2} g \cos \alpha(t), \\
& \xi_{425}=-\frac{1}{8}\left(\frac{1}{3} \theta_{0}+\theta_{1}\right) \theta_{1}^{3} g \cos \alpha(t)\left(1+\sin ^{2} \alpha(t)\right), \\
& \xi_{43}=\frac{1}{2} d_{0} \theta_{1} \cos \alpha(t), \xi_{44}=\xi_{441} l_{0}^{3}+\xi_{442} l_{1}^{3}, \\
& \xi_{441}=-d_{1}\left(\frac{1}{3} \theta_{0}+\theta_{1}\right), \xi_{442}=-\frac{1}{4} d_{1} \theta_{1} \sin ^{2} \alpha(t), \\
& \chi_{3}=\frac{1}{3} \theta_{1}, \chi_{4}=-\frac{1}{2} \theta_{1} \cos \alpha(t), \theta_{0}=\frac{\pi \phi_{0}^{2}}{4} \rho, \theta_{1}=\frac{\pi \phi_{1}^{2}}{4} \rho
\end{aligned}
$$

行列 $A(\alpha(t), \beta)$ および $B_{2}(\alpha(t), \beta)$ は，設計変数である $l_{0}, l_{1}$ に関する複雑な非線形関数になっている. よって, 本設計 例に対しては, 文献 ${ }^{(11)(12)}$ の手法は適用できない。本論文で 提案した統合化設計手法は, LMI による 1 回の設計変数の 更新量はその一次近似が有効になる範囲内に制限されるた め, このような場合でも有効である. 